\title{
TOWARD A SILENT SPEECH INTERFACE BASED ON UNSPOKEN SPEECH
}

\author{
Torres-García Alejandro Antonio ${ }^{1}$, Reyes-García Carlos Alberto ${ }^{1}$ and Villaseñor-Pineda Luis ${ }^{1}$ \\ ${ }^{1}$ Computer Sciences Department, National Institute for Astrophysics Optics and Electronics, Luis Enrique Erro \# 1, \\ Tonantzintla, México \\ alejandro.torres@ccc.inaoep.mx, \{kargaxxi,villasen\}@inaoep.mx
}

\begin{abstract}
Keywords: Silent Speech Interfaces (SSI), Electroencephalograms (EEG), Unspoken Speech, Discrete Wavelet Transform (DWT), Classification.

Abstract: This work aims to interpret the EEG signals associated with actions to imagine the pronunciation of words that belong to a reduced vocabulary without moving the articulatory muscles and without uttering any audible sound (unspoken speech). Specifically, the vocabulary reflects movements to control the cursor on the computer. We have recorded EEG signals from 21 subjects using a markers based basic protocol. The discrete wavelet transform (DWT) is used to extract features from the delimited windows, and a subset of them with frequency ranges below $32 \mathrm{~Hz}$ is further selected. These subsets are used to train four classifiers: Naive Bayes (NB), Random Forests (RF), support vector machine (SVM), and Bagging-RF. The results are still preliminary but encouraging because the accuracy rates are above $20 \%$, i.e. up to chance for five classes. The implementation process as well as some experiments with their corresponding results are shown.
\end{abstract}

\section{INTRODUCTION}

Oral communication is the natural way in which humans interact. However, in some circumstances, it is not possible to emit an intelligible acoustic signal, or it is desired to communicate without making sounds. In these conditions systems that enable spoken communication in the absence of an acoustic signal are desirable. This kind of systems are part of a recent research area called silent speech interfaces (SSI).

Among the SSI technologies described by Denby (Denby et al., 2010), of particular interest are those that use EEG because they are non invasive, relatively simple, economical, and insensitive to environments with large amounts of audible noise. Particularly those associated with unspoken speech, also referred as internal or imagined speech.

Works using unspoken speech can be divided into two approaches: by words and by syllables. The first approach is followed in (Porbadnigk, 2008, Suppes et al., 1997; Wester and Schultz, 2006). While in (Brigham and Kumar, 2010; DaSalla et al., 2009, D'Zmura et al., 2009) only syllables are treated. In the specific case of works that explore words, where this study falls, the following problems had been identified. In (Suppes et al., 1997) was presented a prototypes based method that is not unsuitable for real-time processing. While in (Porbadnigk, 2008, Wester and Schultz, 2006) it is assumed that the extracted features can be recognized with existing models for common speech recognition, nonetheless speech acoustic signal and EEG signals have very different characteristics. In the words approach, the most recent work described in (Porbadnigk, 2008) uses a 5 words vocabulary, EEG signals from seven subjects were recorded, and each word of vocabulary was repeated 20 times.

This research aims to interpret the EEG signals associated with unspoken speech. Specifically, it aims to interpret the signals to recognize five unspoken words of the Spanish language: "arriba", "abajo", "izquierda", "derecha", and "seleccionar", which are repeated 33 times by each subject. They were chosen because with them it could be possible to control a computer screen cursor.

\section{METHODOLOGY}

The stages of proposed methodology are the following: EEG signal adquisition, EEG signal enhancement (Pre-processing), feature extraction, feature selection, and classification.

For EEG signal acquisition an EMOTIV kit is used. This kit is wireless and has fourteen high resolu- 
tion electrodes (channels) whose sampling frequency is $128 \mathrm{~Hz}$.

\subsection{Brain Signals Acquisition}

In this stage the EEG kit is used to acquire the brain signals. According to the Geschwind-Wernicke model, EEG signals related to speech production come from specific areas in the left side of the brain (Geschwind, 1972). Particularly, channels F7, FC5, T7 and P7 are of interest because they are the nearest to Geschwind-Wernicke's model areas.

Moreover, a basic protocol is used to acquire EEG signals from each subject, a mouse is used to send markers to the EEG signals acquisition software, to delimit the start and end of imagining the pronunciation of the words (see Figure 1). A set of samples between both markers is called window or instance.

Considering that, it is known in what part of the EEG signal the patterns associated with the imagination of the word pronunciation should be searched.

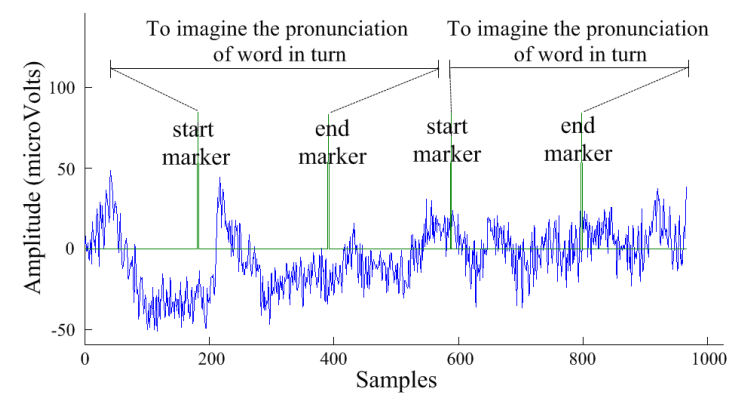

Figure 1: EEG signal from F7 channel that it belong subject S1 while he imagines word pronunciation "abajo" following the data acquisition protocol.

\subsection{Pre-processing}

In this stage, the EEG signals obtained from the channels of interest (F7, FC5, T7 y P7) are filtered using a finite impulse response (FIR) band-pass filter at the range 4 to $25 \mathrm{~Hz}$.

It is noteworthy that, similarly to conventional speech, the duration of the unspoken speech's windows for each word is variable, for one subject as well as for different subjects. Thus, it is necessary to establish an equal size for all windows.

At the end of this stage windows with 256 samples and a frequency range between 4 and $25 \mathrm{~Hz}$. are kept and used for the creation of the experimental data base. Windows lower than 256 samples are completed with zeroes, and those with more than 256 samples are discarded.

\subsection{Feature Extraction}

In (Lotte et al., 2007) it is mentioned that the features to be used in BCI are not stationary and contain time information, which makes necessary an adequate representation. The discrete wavelet transform (DWT) provides a highly efficient wavelet representation by restricting the variation in translation and scale, usually to powers of two.

In consequence, in this work the discrete wavelet transform (DWT) with six decomposition levels is applied, using a second order Daubechies (db2) as mother wavelet function. With this, a vector with 269 wavelet coefficients is obtained for each window in each of the interest channels. Subsequently, the coefficients in the same time interval that belong to the four interest channels are concatenated following this order F7-FC5-T7-P7. At the end of this stage is obtained a vector with 1076 features, and its corresponding class label is obtained.

\subsection{Feature Selection}

The feature selection problem implies to select a minimum subset, with $M$ features, $S=\left(S_{1}, \ldots, S_{M}\right)$ from original feature set $F=\left(F_{1}, \cdots, F_{N}\right)$, where $M \leq N$ and $S \subseteq F$, so that the feature space is optimally reduced and the classification performance is maintained, improved or not significantly degraded.

At this stage, the subset of features greater than $25 \mathrm{~Hz}$. is discarded. Therefore, the feature subset selected consists of the detail coefficients D2 to D6 and the approximation A6 which reduces the dimension of the feature vectors, and at the same time reduces the impact of the curse of dimensionality in the classification stage. With this, each window of each channel is represented with 140 wavelet coefficients. Then, the DWT coefficients of windows in the same time interval were concatenated as in the feature extraction stage.

\subsection{Classification}

In this work the following three classifiers are trained and tested: Support Vector Machines (SVM), Random Forests (RF) and Naive Bayes (NB). After evaluating the individual classifiers, the classifier with the higher accuracy percentage is selected to use it as the base classifier in Bagging ensamble. 


\section{EXPERIMENTATION AND RESULTS}

\subsection{Preliminary Experiments}

Preliminary experiments consisted in training and testing the three described classifiers (NB, RF, and $\mathrm{SVM}$ ) with the EEG signals recorded from three subjects (S1, S2 and S3). Previously, complete and reduced feature vectors are obtained. These experiments aim to evaluate the convenience of using the complete or reduced vectors, and select the classifier to be used as a basis for the Bagging classification. For this purpose, the measure to evaluate the classifiers is accuracy. The classification accuracy is obtained through 10-fold cross validation.

The accuracy percentage for each of the three classifiers using the complete feature vectors are in table 1.

Table 1: Accuracy percentages obtained for the classifiers using the complete feature vectors (1076 features).

\begin{tabular}{|l|c|c|c|}
\cline { 2 - 4 } \multicolumn{1}{c|}{} & \multicolumn{3}{c|}{ Accuracy } \\
\hline Subject & NB & RF & SVM \\
\hline S1 & 23.35 & 24.08 & 23.35 \\
\hline S2 & 17.09 & 31.63 & 24.78 \\
\hline S3 & 35.75 & 41.21 & 18.18 \\
\hline
\end{tabular}

Table 2 presents the accuracy percentage for each of the three classifiers using the reduced feature vectors.

Table 2: Accuracy percentages obtained for the classifiers using the reduced features vectors (540 features).

\begin{tabular}{|l|c|c|c|}
\cline { 2 - 4 } \multicolumn{1}{c|}{} & \multicolumn{3}{c|}{ Accuracy } \\
\hline Subject & NB & RF & SVM \\
\hline S1 & 24.08 & 43.78 & 21.9 \\
\hline S2 & 18.8 & 38.46 & 21.37 \\
\hline S3 & 33.94 & 43.64 & 19.39 \\
\hline
\end{tabular}

The results described in tables 1 and 2 show that, generally, better results are obtained when using the reduced feature vectors than when using complete vectors. Nonetheless, in occasions when better results are obtained using the whole vectors, the improvement is not significant.

In addition, the tables 1 and 2 show that the classifier which obtains the best accuracy percentages is $\mathrm{RF}$, so it was selected to be used for the following experiments as the base classifier for Bagging. From here, this ensemble will be denoted as Bagging-RF.

\subsection{Experiment with the whole corpus}

In these experiments participated 21 right-handed subjects (S1-S21) to collect a corpus of data. From each of them 33 instances of each of the five imagined words were recorded. However, those instances with more than 256 samples (two seconds long), are discarded in the experimental phase. After that selection process, the remaining instances pass by all methodology stages.

In the first experiment the instances from each of the twenty-one subjects (S1-S21) are separately utilized for training and testing the four evaluated classifiers (RF, SVM, NB, Bagging-RF). The accuracy percentages are obtained using 10-folds cross validation. In figure 2 these percentages are shown.

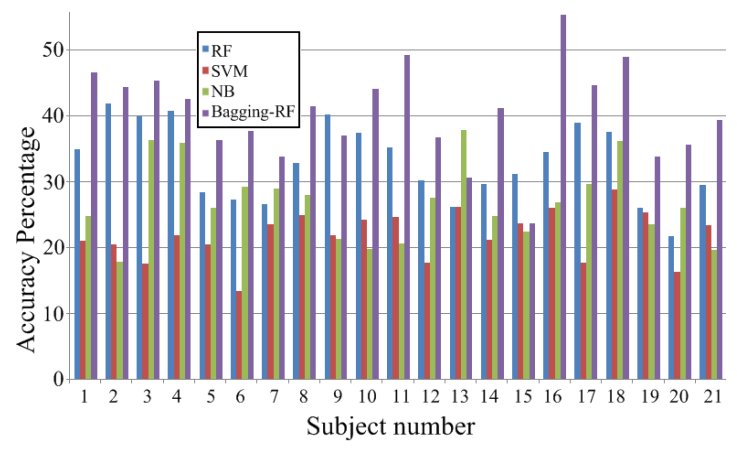

Figure 2: Accuracy percentages for each classifier obtained after 10 -folds cross validation on the data of each subject.

Figure 2 shows that, generally, the accuracy percentages obtained by the four classifiers are above chance for five classes, which is $20 \%$. This accuracy rate is taken as a lower bound because, according to (Dietterich, 2000), "an accurate classifier is one that has an error rate better than chance at the stage of generalization (testing)". Furthermore, figure 2 shows that, according to accuracy percentages, the best classifier is Bagging-RF and the worst is SVM. Also, it is important to note that, for all subjects both RF and Bagging-RF are kept above chance for the five classes.

Further on, results at word level obtained by RF and Bagging-RF, utilizing the F-measure, are next presented. For each subject's dataset, the classifiers calculate a F-measure value for each of the words.

Figure 3 shows the average F-measure obtained by $\mathrm{RF}$ and Bagging-RF for each of the words. In the case of RF, the words order according to the f-measure from high to low, is: "arriba", "izquierda", "seleccionar", "abajo", and "derecha". While, in the case of Bagging-RF according to the same F-measure the order is: "seleccionar", "arriba" , "derecha", "abajo", and "izquierda". 


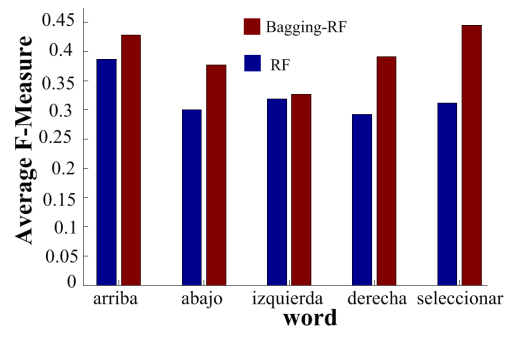

Figure 3: Graph of average f-measure for each word obtained for RF and Bagging RF using 10-folds cross validation on the subject's data.

It is important to note that, the words "seleccionar" and "arriba" classified by Bagging-RF have a F-measure above 0.4, which is twice bigger than random.

Last, it is worth to mention that in the presented work the results obtained are relatively comparable to state of the art similar works, like those reported in (Porbadnigk, 2008) where the classification was evaluated only based in accuracy, reporting $45.95 \%$ for five words. This comparison is mentioned considering the differences described in section 1

\section{CONCLUSIONS AND FUTURE WORK}

The acoustic speech signal and the EEG signals have different features, which makes them naturally dissimilar. In consequence, we explored an alternative processing and classification approach to treat the EEG signals, in particular those related to unspoken speech. Indeed, the problem of interpreting unspoken speech is still far to be solved. However, from our experiments we obtained evidence to affirm that the EEG signals actually carry useful information to allow the classification of unspoken words. We conclude this based on the percentages of accuracy in the classification for the four classifiers, which, are above chance for five classes (see figure 2). Our results and experimental procedures are consistent with those reported in the state of the art, because: we performed experiments with more than one classifier, we explored a language different to English, we used a reduced vocabulary with more semantic meaning, and we worked with features obtained by a feature selection approach instead a dimensionality reduction approach. The average f-measure was below the percentages due to chance for five classes.

To improve the reported results we propose to explore how to utilize and compare all windows regardless their size. We propose to apply independent com- ponent analysis (ICA) and assess each independent component using the Hurst's coefficient to eliminate some artifacts as blinks and heartbeats. To select another wavelet family also could help. We also plan to test another EEG signal representation and combine them with the DWT coefficients. Finally, it is still possible to use hybrid intelligent systems, and others ensemble schemes to improve classification results.

\section{ACKNOWLEDGEMENTS}

This work was done under partial support of CONACyT (scholarship \# 234705), and INAOE.

\section{REFERENCES}

Brigham, K. and Kumar, B. (2010). Imagined Speech Classification with EEG Signals for Silent Communication: A Preliminary Investigation into Synthetic Telepathy. In Bioinformatics and Biomedical Engineering (iCBBE), 2010 4th International Conference on, pages 1-4. IEEE.

DaSalla, C. S., Kambara, H., Koike, Y., and Sato, M. (2009). Spatial filtering and single-trial classification of EEG during vowel speech imagery. In $i$-CREATe '09: Proceedings of the 3rd International Convention on Rehabilitation Engineering \& Assistive Technology, pages 1-4, New York, NY, USA. ACM.

Denby, B., Schultz, T., Honda, K., Hueber, T., Gilbert, J., and Brumberg, J. (2010). Silent speech interfaces. Speech Communication, 52(4):270-287.

Dietterich, T. (2000). Ensemble methods in machine learning. Multiple classifier systems, pages 1-15.

D'Zmura, M., Deng, S., Lappas, T., Thorpe, S., and Srinivasan, R. (2009). Toward EEG sensing of imagined speech. Human-Computer Interaction. New Trends, pages $40-48$.

Geschwind, N. (1972). Language and the brain. Scientific American.

Lotte, F., Congedo, M., Lécuyer, A., Lamarche, F., and Arnald, B. (2007). A review of classication algorithms for EEG-based brain-computer interfaces. Journal of Neural Engineering, 4:r1-r13.

Porbadnigk, A. (2008). EEG-based Speech Recognition: Impact of Experimental Design on Performance. Master's thesis, Institut für Theoretische Informatik Universität Karlsruhe (TH), Karlsruhe, Germany.

Suppes, P., Lu, Z., and Han, B. (1997). Brain wave recognition of words. Proceedings of the National Academy of Sciences of the United States of America, 94(26): 14965.

Wester, M. and Schultz, T. (2006). Unspoken Speech Speech Recognition Based On Electroencephalography. Master's thesis, Institut für Theoretische Informatik Universität Karlsruhe (TH), Karlsruhe, Germany. 